\title{
Composite Score
}

National Cancer Institute

\section{Source}

National Cancer Institute. Composite Score. NCI Thesaurus. Code C121197.

A single value that is calculated from multiple scores. This composite score can then be used as a single variable in further analysis and comparison. 